\title{
Association of Food Intolerance with Coronary Artery Disease
}

\author{
Angeliki Tsantsaridou ${ }^{1}$, Vissarion - Panagiotis Papathanasiou ${ }^{2}$, Gerasimos Dimisianos ${ }^{3,{ }^{*}}$ \\ ${ }^{1}$ Department of Cardiovascular and Thoracic Surgery, Larissa University Hospital, Larissa, Greece \\ ${ }^{2} \mathrm{MSc}$ Statistician, Levadia, Greece \\ ${ }^{3}$ Molecular Biologist, PhD, Larissa, Greece \\ *Corresponding author: gdimisianos@hotmail.com
}

Received November 12, 2018; Revised January 07, 2019; Accepted January 25, 2019

\begin{abstract}
Background: Hippocrates wisely said that «your food be your medicine and that nature is the doctor of diseases». In the Holy Scriptures the «clean from the unclean foods» are separated. In this pilot study, we tried to uncover any intolerances to foods of patients with coronary artery disease. Aim: The purpose of this trial was to evaluate any food categories that stimulate the immune system of patients with coronary heart disease by creating a vicious circle of inflammation, including atherogenesis. The analysis will contribute to possibly understand patients with coronary artery disease, the aetiopathogenesis of the underlying disease and implement the appropriate therapeutic nutrition. Materials and methods: The presence of IgG antibodies were detected using a kit (Golden test) with ELISA method in 63 patients and 12 healthy individuals. Results: The analysis revealed that more frequent intolerances were in banana, milk products and eggs. The tests showed statistical differences for banana and milk among the two groups. Conclusions: Although most patients had an intolerance in bananas, eggs and milk, this work does not verify the association of food intolerances with the etiology of coronary artery disease.
\end{abstract}

Keywords: immune system, $\operatorname{IgG}$, coronary artery disease, food intolerance

Cite This Article: Angeliki Tsantsaridou, Vissarion - Panagiotis Papathanasiou, and Gerasimos Dimisianos, "Association of Food Intolerance with Coronary Artery Disease." Journal of Food and Nutrition Research, vol. 7, no. 1 (2019): 71-81. doi: 10.12691/jfnr-7-1-9.

\section{Introduction}

There is a biochemical and immunological basis of atherosclerosis and vascular inflammation. Atherosclerosis is a chronic inflammatory disease of the vascular wall. The atheroma is a response of the immune system to exogenous and endogenous antigens, causing damage to the endothelium. Immunity receptors recognize dangerous molecules and antibodies which are generated. The IgG is the main immunoglobulin in the blood circulation. These antibodies are detected in atherosclerotic lesions and their title has been associated with cardiovascular risk. Antibodies and products of oxidation and apoptosis all contribute to the formation of the atherosclerotic plaque. [1]

More often, patients with hyperlipidemia present higher body mass index, diabetes mellitus and hypertension. Also IgG antibody titer against micro-organisms (anti-HSV2, anti-CMV) as measured with the ELISA method are statistically higher. The immune response ( $\operatorname{IgG})$ supports the correlation of infection/inflammation with atherosclerosis. [2,3]

The connection between inflammation, obesity, hypertension and coronary artery disease remains unclear. In all these parameters, IgG antibody and CRP (C-reactive protein) production, enzyme dysfunction, are "waterfall" reactions. Immunoglobulin levels are biomarkers of humoral defense and oxidative stress. The immune mechanisms are implicated in chronic inflammatory response, hypertension, vascular dysfunction and deterioration of organs. $[4,5,6]$

Inflammation is a key feature of atherosclerosis. Elevated levels of white blood cells (eosinophils, monocytes, neutrophils, lymphocytes) and the CRP, the immune responses herald adverse cardiac outcomes, endothelial activation, growth and rupture of the plaque.

Chronic subclinical inflammation plays a leading role in the initiation and establishment of diabetes, a predisposing factor of coronary artery disease. $[7,8]$

Chronic inflammation and intense promoting immune responses, are commented in one case of a patient with a history of chronic hepatitis C, AIDS, angioplasty, and irritable bowel syndrome. [9]

Many patients with coronary artery disease suffer from malignancies, autoimmune disorders, infections which alter the inflammatory procedures and immune system. In prolonged stress situations, microbial or other loads, cytokines (molecules coming from endothelial cells, macrophages, lymphocytes), intercellular mediators are released. Differentiated B cells or other cells induce C-reactive protein production. [10]

The inflammatory markers, such as CRP, are associated with dyslipidemias and cardiovascular risk. The CRP is 
present in atherosclerotic plaques and contributes to their development and their volatility. [11,12]

The arterial lesions are caused by factors, such as genes, vascular wall injuries, tobacco, sugar, hypertension. Aging and the ability to repair damages have been linked to inflammatory processes in various stimuli (germs, elevated cholesterol, etc.) and eventually lead to dysfunction of tissues and organs. [13]

The inadequacy of the anti-oxidant enzyme systems, oxidative stress and the creation of active oxygen radicals cause dysfunction of mitochondria, genetic changes leading to impaired glycolysis, abnormal fat metabolism, glucose intolerance (: metabolic syndrome), promoting the accumulation of fats and atherosclerosis. The DNA of vascular cells is vulnerable to damage. Gene modifications affect cellular behavior, development, aging and death. All these might contribute to atheromatic plaque formation, rupture and myocardial infarction. [14]

Atherosclerotic lesions include inflammation, infiltration of macrophages and various proteins. [15] The endothelial dysfunction leads to vasoconstriction, ischemia and clot formation. Substances derived from platelets, lead to vasoconstriction and hyperplasia of the vessel wall (an indicator of early atherosclerosis). [16,17,18]

Substances that are created in the gut and brain, can stimulate mast cells to release mediators (interleukins, etc.). Neurological and immunological malfunctions are created which lead to increased vascular permeability and endothelial growth factor secretion by mast cells. These cells are involved in innate and adaptive immunity and inflammation. [19]

The cause of most vascular diseases is the dysfunction of the endothelium. The endothelial cells show an ability to transition from a noninvasive to proliferative state. This process is driven by various factors (vascular endothelial factor etc.) and metabolic "switches". Disturbances in the metabolism of vascular system affect and are affected by metabolism of other cells. Metabolic changes in diabetics, cancer patients and other diseases are related to metabolic changes of endothelial cells. Hypoxia, vascular endothelial growth factor, oxidative stress are involved in atherosclerosis. [20,21,22]

The hyperlipidemias is one of the main risk factors for diabetes, aging, atherosclerosis. The accumulation of lipids (cholesterol, triglycerides) in the vascular endothelium, activates leukocytes for the production of cytokines and the recruitment of macrophages. On the other hand the macrophages secrete a vascular endothelial growth factor that mediates angiogenesis and inflammatory reactions. Important mediators of inflammation in atherosclerosis are: C-reactive protein, amyloid, interleukins 1 and 6 , metalloproteases, TNF (tumor necrosis factor) etc. Inflammation indicators characterize the situation of vascular wall and these are involved in atherogenesis. Hypertension accelerates the atherosclerotic inflammatory process. In summary the inflammation with the mediators because of immune stimulation, are implicated in hypertension and cardiovascular disease. [23,24,25]

Inflammatory reactions of the immune system could trigger processes of arteriosclerosis. Within us inhabit numerous friendly and hostile microorganisms. Changes in their ratio cause an imbalance of oxidants and antioxidants factors and metabolic disorders, obesity, diabetes, arteriosclerosis. [26]

\subsection{Inflammation and Dietary Habits}

We emphasize in the importance of food and its ingredients, the breakdown molecules and the absorption capacity of the human body. They can protect or harm our health. The side effects of food consumption are determined by immunological and other reactions. The food antigens can be their microbial load, their proteins, the mixture of antibiotics, chemicals, pigments, preservatives. [27,28]

The antibodies are immunoglobulins ( $\mathrm{IgG}$ ) which are produced by $\mathrm{B}$ cells and bind to the antigens in order to inactivate them. When the antigen is linked to a cell (e.g. bacteria), this is destroyed through activation of complement and the phagocytosis by macrophages.

In hypertension elevated levels of $\operatorname{IgG}$ and $\operatorname{IgM}$ antibodies are observed. These antibodies target receptors and ion channels $\mathrm{Ca}++, \mathrm{Na}+$, and water channels. All play an important role in regulating blood pressure (a risk factor of arteriosclerosis).

The antibody-antigen binding to the surface of endogenous cells can lead to their destruction. The immune complexes deposited in various tissues cause inflammation including vasculitis, hypertension, diabetes and autoimmune disorders. [29]

The metabolism is associated with the immune system. The lipolytic stages involve the removal of lipid overload with blood lipoproteins. Antibodies in autoimmune hyperlipidemias prevent these stages. [30,31]

Not a lot of research exists in the role of dietary factors and food intolerance in the pathogenesis of cardiovascular diseases. It is known that eating habits/disorders alter the risk factors of coronary artery disease. The metabolic syndrome is an example of such a relation. Food intolerance includes immune reactions of the organism against certain foods. It is not a food allergy, although these two may coexist. Dairy products, cereals containing gluten and legumes are mainly implicated. Symptoms such as irritable bowel, skin manifestations (eczema, psoriasis etc.), respiratory events (asthma, etc.) are created. It is delayed hypersensitivity reaction mediated by cells and involves the production of non- $\operatorname{IgE}$ antibodies. Eventually a wide range of diseases appear. After the primary exposure to "toxic" stimulus, activating defense mechanisms (antibodies, cytokines, lymphocyte reactions etc.) follow and finally food intolerance is "installed" along with physical and the neuropsychological events. $[32,33,34]$

$15-20 \%$ of the general population shows intolerance to foods, due to their "pharmacological" ingredients, the lack of digestive enzymes and transfer substances, as well as the microbial load of food. Usually they create gastrointestinal symptoms. In some individuals, food and their proteins are treated as foreign bodies, stimulating a chain of inflammatory reactions in the digestive mucosa. The defensive system is creating antibodies against the "invaders" (food and antigens). Mast cells (basophils connective tissue cells of the blood), eosinophils, lymphocytes migrate and proliferate (lymphatic mucosal 
hyperplasia). Epithelial cells are also involved in the inflammatory process. Ultimately, immune tolerance evolves. [35,36]

In an observational study (12 years) of 499 patients with systemic lupus erythematosus disease, they measured the IgG antibody levels against anti-apolipoprotein a-1 and their association with inflammatory disease activity, the risk of developing atherosclerosis and cardiovascular events, mortality. Patients with active disease (27\%) had higher levels of antibodies. Their association with mortality and morbidity was not substantiated. [37]

In one experiment, they introduced in mice intravenously endotoxins and they measured $\operatorname{IgG}$ antibodies against apolipoprotein A1 (apoA1 anti-IgG). This resulted in creation of unstable atherosclerotic plaque, ischemia and myocardial infarction. The inflammation seemed to be promoted through immune receptors. [38]

Antigens are toxic substances and microorganisms in food. Excluding the guilty foods from the diet, improves the symptoms of patients. Inoculation of microbial factors induce the production of cytokines and the production of immunoglobulins $\operatorname{IgA}$ and $\mathrm{IgG}$. Inflammatory reactions participate in atherogenesis, the destabilization of the plaque and heart disease (hypertension, atrial fibrillation, angina, heart attack and heart failure). [39,40]

Dietary factors not tolerated by the patient interact with host defense system and result in inflammatory processes. Then chronic diseases related to food appear, including arteriosclerosis, metabolic syndrome, Crohn disease, ulcerative colitis. The diet, which is adjusted for every individual, in accordance with the result of intolerance leads to immune homeostasis, treatment of diseases and good overall health. The pathogenic mechanisms of atherosclerosis are associated with the lipid metabolism and inflammation. Inflammation and metabolic disorders are connected with food intolerance. [41,42]

The mechanisms involved in food intolerance, include immunological responses, lack of enzymes, food ingredients (pharmacological, irritant and toxic substances), oxidative stress. The inadequacy of the anti-oxidant enzyme systems, oxidative stress and the creation of active oxygen radicals cause dysfunction of mitochondria, DNA and fat damages. The cellular aging and organ malfunctioning is associated with oxidative stress. The dysfunction of the endothelium due to oxidative stress, leads to deregulation of vascular tone, decreased angiogenesis, hypertension, cell migration, adhesion, thrombosis, atherosclerosis development, poor perfusion, inflammation, cardiovascular disease and even cancer. The increased oxidative stress increased by aging, leads to destruction of the proteins, lipids and nucleic acids. Several studies have demonstrated a relationship of these disorders, causing endothelial dysfunction, hypertension, atherosclerosis, ischemia infarction, heart failure. [43]

The dietary factor is perceived as a daily stressful load. Nutrition is the connection between immune activation and inflammation, resulting in illness, the onset of chronic diseases (: metabolic syndrome, atherosclerosis, ulcerative colitis, Crohn disease, etc.).The cellular immunity, especially neutrophils reactions and immunoglobulins Ig production are processed. A "junk" food in the gastrointestinal system causes lymphocytic infiltration of the intestinal epithelium, increased spacing between the villi, cracks. [44,45,46]
The gastrointestinal mucosa contains eosinophils, lymphocytes, mast cells that react food and bacterial allergens. Proteins in food, usually, serve as main antigens, which cause the most allergic reactions to gastrointestinal tract. The gut sites, play a vital role in preventing diseases of endogenous organs: 1) is a physical barrier, preventing the alien invasion, 2) specialized immune cells, including macrophages, are present there which phagocytose bacteria and other antigens 3) IgA antibodies are released, which fight the microbes in the lumen of the gut, 4) promoting the binding of cells with antigen to the antibodies (intolerance reaction) 5) with T-cells the gastrointestinal system modulates the immune response. Chronic inflammation in the intestines leads to damage to the natural barrier of the intestine to the outer environment, resulting in the infiltration of inflammatory antigens in its space. Antigens of foods trigger the IgG and IgM antibody production. Especially desserts, dairy products and meat cause symptoms of intolerance such as diarrhea, abdominal pain. $[47,48,49]$

The food antigens react with tissue proteins of the host, causing degenerative and autoimmune diseases, such as diabetes, atherosclerosis, inflammation, neurological deterioration, and hypertension. The IgG, IgA and IgM antibodies produced against the foreign bodies, have a strong impact on cellular dysfunction, inflammation of tissues (and of the vascular wall), of organs, a process associated with diabetes, the metabolic syndrome, renal insufficiency and aging. Intolerance to foods leads to a deficiency of micronutrients and organ damage. Dietary changes prevent cardiovascular events. [50]

The activation of the immune system by food antigens and alterations of the endogenous microbial flora, all lead to functional changes of gastrointestinal system and a chain of pathological situations such as Crohn disease, colitis, atherosclerosis, cancer, autoimmune disorders. Celiac disease is prevalent in $1 \%$ of the Western general population. It is autoimmune disorder after exposure to gluten (wheat protein). When the small intestine is exposed to it, the CD T-cells are presented, cytokines are released and eventually the intestinal mucosa changes histologically. Systemic manifestations occur (abdominal pain, diarrhea, anemia, arthritis, dermatitis, cancers, lymphomas, autoimmune diseases, etc.). Gluten does not break down nor is it absorbed properly. Gliadin is a product of enzymatic cleavage of wheat, toxic (: antigen) for individuals with intolerance. Defense mechanisms and inflammatory procedures are stimulated. The intestinal microbial load changes. These individuals should avoid barley, wheat, rye, but can eat rice, quinoa, and corn. The diagnosis of food intolerance in patients with celiac disease was documented by mucosal duodenal biopsy and the identification of IgA, IgG antibodies (ELISA method) to gliadin and transglutaminase. The intolerance to food of these patients with celiac disease is due to lower activity of one or many enzymes. [51,52,53]

The reactions to food and their proteins induce chronic inflammation in the intestinal mucosa. The two mechanisms, allergy and intolerance, can co-exist. Some proteins primarily animal (dairy, meat) hardly digested in the gastrointestinal tract, are recognized as allergens. Their molecules are large. Immunological and other mechanisms control their penetration through the gut. If 
the digestive enzymes which are secreted and the $\operatorname{IgA}$ antibodies which are produced (joined with antigens) do not suffice, the permeability of intracellular and extracellular macromolecules is increased, so the gastrointestinal infections. All damage the gastrointestinal epithelium and allow proteins to penetrate into the circulation and activate the immune system. Consequently the food intolerance and its symptoms are established. The inflammation degrades the intestinal mucosa and macromolecules permeate the gastrointestinal epithelia and are recognized by the immune system. Antibodies are produced (IgG etc.) and bind to antigens. These complexes (IgG-Ag) are deposited to vessel endothelium and initiate inflammation (atheroma). There is no barrier to the external environment and the nutrients are not properly absorbed. To eliminate malnutrition, dehydration etc., the individuals should remove the food antigens. Probiotics and Omega- 3 fatty acids protect the gut from inflammation. It is estimated that one fifth of the population have negative reactions to food. The reactions vary from allergies (anaphylaxis, shock and death) to food intolerances. [54,55]

Serological markers of intolerance to foods are the IgA and IgG antibodies. Food antigens are microbial and biochemical. The IgA and IgG are highly elevated in patients with autoimmune disorders e.g. Crohn disease. [56]

Lack of digestive enzymes result in malabsorption and intolerance to foods. Most adults have a reduced activity or deficiency of the lactase enzyme, resulting in gastrointestinal discomfort. $30-40 \%$ of the population do not absorb sugars. [57]

Intolerance to foods leads to type I hypersensitivity reactions. These are influenced by genetic factors, the nature of the antigen and the bacterial infections. Interleukins and antibodies are produced. The antibodies bind to receptors and mediators are released, which create tissue damage. The food intolerance test by ELISA method (enzyme linked immunosorbent assay) detects the percentage of circulating IgG immunoglobulin, which joins each food category (intolerance). Exclusion of antigenic foods from the diet, administration of steroids and immunosuppressants (cyclosporine, azathioprine) are effective as therapy. Data markers of apoptosis after dietary change are recorded. These agree with the correction of immunological disorders. [58,59]

Food intolerance is associated with the predisposing risk factors (e.g. obesity) of coronary artery disease. In one study, when they altered the diets of the patients with high body mass index, then their body weight became normal. The fat metabolism is associated with the immune system. The IgG antibody inhibits lipolysis of triglycerides, because it reacts with a substance necessary for the activity of lipoprotein lipase. The hypertriglyceridemia is due to antibodies (IgG) against lipases. [60-64]

Lipid disorders in patients with systemic lupus erythematosus are associated with long-term inflammatory autoimmune disorder that affects multiple systems. The IgG anti-cardiolipin antibodies affect lipid levels. More specifically, patients had significantly lower HDL-cholesterol and greater incidence of cerebral, thromboembolic and cardiovascular events. These are connected with the circulation of IgG and IgM anticardiolipin antibodies. [65,66]

The wheat gluten has been implicated in gastrointestinal symptoms, celiac disease and irritable bowel syndrome.
People with this syndrome have increased IgE, IgG levels. The balance of solid-liquid-gas in the digestive tract is affected by the dietary load, the absorption capacity and the microbial flora. Restriction of the food amount, of sugars and carbohydrates improves symptoms. The microbial flora of the gut plays a vital role in homeostasis, in health and disease pathogenesis. It protects against pathogens, affects the development of immune system, participates in the metabolism and nourishment. Changes in microflora by nutrition and probiotics affect inflammatory responses and immunological phenomena. The food categories and age affect the appearance of food intolerance and disease. Food intolerance in patients with an irritable bowel is characterized by an increased synthesis of IgG antibodies and inadequate production of anti-inflammatory factors, cytokines, etc. [67,68]

The metabolic syndrome is a cardiovascular risk factor. It includes central obesity, insulin resistance and lipid disorders. Cardiovascular disease appears to be an inflammatory procedure, leading to damaged endothelium. Patients with celiac disease have an increased risk of cardiovascular disease. Inflammation, cytokines and CRP are biomarkers of metabolic syndrome and cardiovascular risk. In celiac disease, the stimulation of the immune system leads to the release of inflammatory factors, which may affect the atherogenesis. Changes in microbial intestinal flora have been related to diet, autoimmune reactions, inflammation. Changes in gastrointestinal microflora are involved in the pathogenesis of metabolic disorders, obesity, diabetes and atherosclerosis. Patients with celiac disease have an increased body mass index. This factor leads faster to the formation of atheromas. Scientists studied the carotid intima thickness (: atherosclerotic changes index) in patients with celiac disease. Compared with the control group, there was a significant increase. A gluten-free diet resulted in a significant decrease in the thickness of the intima and inflammation, reduced obesity and insulin resistance, and an increase in HDL-cholesterol. [69,70]

Hypertension is a predisposing risk factor of arteriosclerosis. The circulating $\operatorname{IgG}$ and $\operatorname{IgM}$ antibody levels are elevated in hypertensive patients. These antibodies target receptors and ion channels (e.g. $\mathrm{Ca}++$ ), which play an important role in regulating blood pressure. Antibodies determine hypertension! The antibody-antigen complex binds to the surface of endogenous cells and can lead to cellular destruction. The immune complexes are deposited in the tissues causing inflammation, including of vasculitis. Elevated levels of $\mathrm{Ca}++$ ions in smooth muscle cells involve increased vascular resistance and hypertension. Elevated $\operatorname{IgG}$ and $\operatorname{IgA}$ antibody titers were spotted in hypertensive patients. Studies have detected an increase of $\operatorname{IgG}$ anti-endothelial and $\operatorname{IgM}$ antibodies floating in blood circulation. These contribute to endothelial dysfunction, hypertension. Hypertension causes oxidative stress in the vascular wall. [71]

\section{Materials and Methods}

Food intolerance of patients with coronary artery disease was checked by simple blood donation and the use of the ready-made commercial kits Food Detective/Golden 
test (Cambridge Nutritional Sciences). The sole criterion for inclusion of patients in the study was the presence of coronary artery disease based on coronary angiography. The severity of the disease was assessed i.e. influenced ejection fraction, strain of the ventricles, the number and extent of atherosclerotic lesions. The classification of the disease was considered as mild, moderate or severe.

Healthy individuals (without coronary artery disease) were also studied (control group). The presence of IgG antibodies for 90 different foods were detected according to the kit manufacturer's instructions. The ELISA (enzyme-linked immunosorbent assay) method was used with the GOLDEN TEST kit as follows: a small amount of blood (serum) and a special solution were added to drive the reaction highlighted with protein/food ingredients. Color (with the help of the enzyme assays) changes (blue color) detect (measuring qualitatively) antibodies.

The disc has posts - rings with food extracts numbered from 1-46. Seats 47 and 48 are to see if the test has been successfully carried out. To be valid the results after the completion of the process, should show position 47 as white (negative result) and post 48 blue (positive result).

List of detectable (Detective) food were: grains, corn, durum wheat, gluten, oats, rice, rye, wheat; nuts and beans almonds, brazil nuts, cashews, cocoa, pistachio, mix legume (pea, lentil, bean), soybean, walnut; meat beef, chicken, lamb, pork; freshwater fish, fish mix (salmon, trout), mixture of shellfish (shrimp, crab, lobster, clams), tuna, white fish mix (COD, haddock, plaice); vegetables broccoli, cabbage, carrot, celery, cucumber, leek, peppers (red, green, yellow), potato; fruits apple, blackcurrant, grapefruit, melon mix (melon, watermelon), olive, orange, lemon, strawberry, and tomato; others were egg (whole), cow's milk, garlic, ginger, mushroom, tea and yeast.

To clarify, the food table is: 1) milk (cow, goat, sheep) 2) a variety of white fish 3) a variety of freshwater fish (salmon, trout) 4) a variety of shrimp, crayfish, sea crab, lobster, mussel, squid 5) a variety of fatty fish (herring, mackerel, tuna, sardines) 6) a variety of lily (asparagus, garlic, leek, onion) 7) a variety of complex (artichoke, lettuce, sunflower seeds) 8) a variety of cucumber, melon, watermelon 9) a variety of carrot, celery, parsley 10) a variety of legumes (beans, peas, chickpea) 11) a variety of crucifer (broccoli, brussels sprouts, cabbage, cauliflower) 12) a variety of beetroot, spinach 13 ) a variety of potato, eggplant, paprika, pepper, chili, sesame, tomato 14) a variety of grapefruit, lemon, mandarin, orange 15) a variety family of apricot, peach, plum 16) a variety of basil, lavender, peppermint, oregano 17) a variety of mushroom, yeast

The analysis will contribute to understand the patients with coronary heart disease, the aetiopathogenesis and implement appropriate therapeutic nutrition.

12 healthy individuals and 63 patients (11 women and 52 men) were studied.

A written consent form for the participation in the study was obtained from all participants, taking into consideration the GDPR Act and the ethics committee of the University.

\section{Results}

In the patient group: the order of severity of food intolerance was as follows: banana, egg, milk, a variety of products such as potato, eggplant, paprika, pepper, chili, sesame, tomato and almond, a variety of legumes and a variety of yeast products.

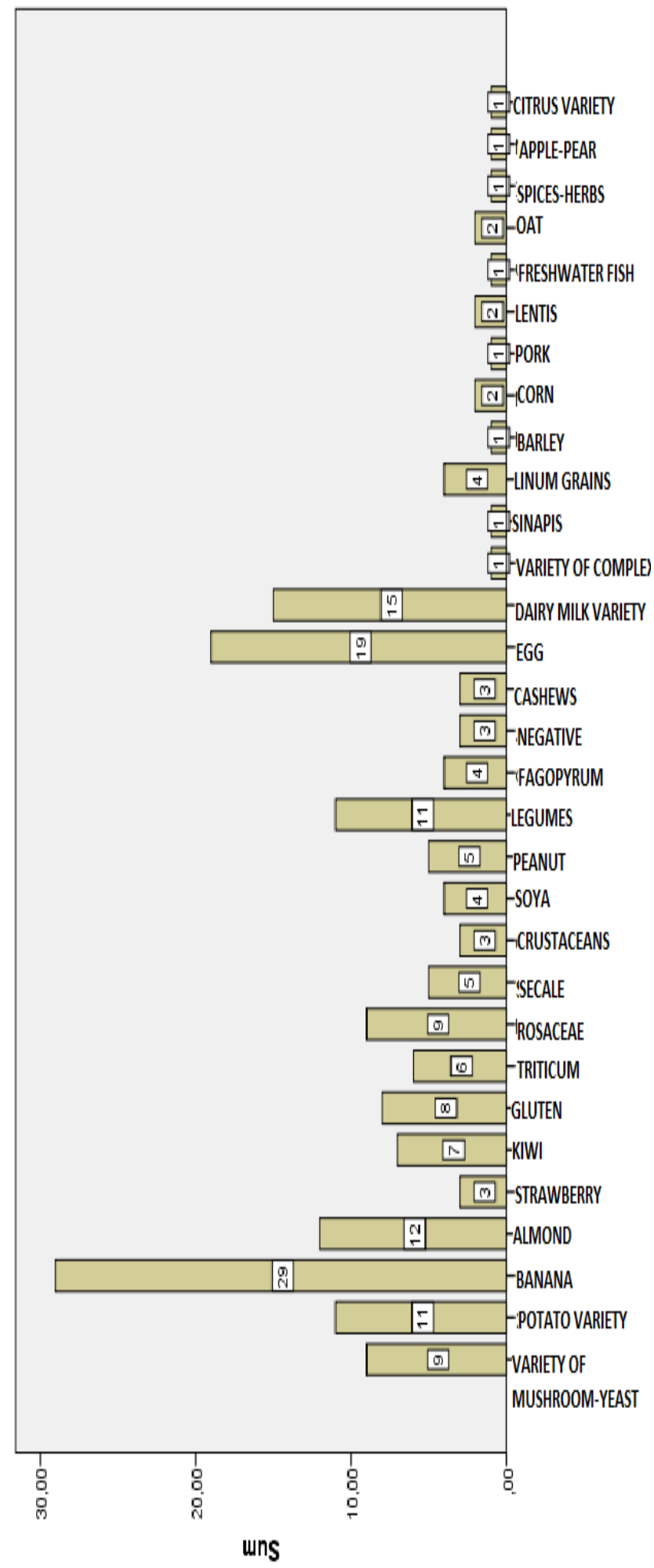

Chart 1. 
The bar chart illustrates the incidences of intolerance in various foods. "Champions" emerge: banana with frequency 29 (45.3\% of individuals with coronary artery disease were diagnosed with an intolerance to bananas) and then follows the egg with 19 (29.6\%) and milk with 11 (17.1\%).

The subsequent conclusions are visual and subjective.

Because the research is qualitative and counts aposteriori food intolerance existence, all intolerances detected either at large or in small frequency are not cause-effect of the disease.

From the bar chart we get the following subjective evidence. The banana appears to be associated with severe forms of the disease. The egg has a strong presence in 3 levels of intensity of the disease with the majority of intolerance in the egg to appear in the mild coronary artery disease. Milk appears in majority in mild forms but does not appear at all in moderate intensity and has a high incidence in severe forms. The other intolerances levels are low and similar to each other and will not refer separately. A particular reference could do in cases of severe disease in which a variety of potato, eggplant, paprika, pepper, chili, sesame, tomato, the almond, the gluten, wheat and legumes have a relatively significant incidence.

The variables "existence disease" and "intolerances" layout and class respectively. It is therefore theoretically and technically impossible to talk about correlation. But we can apply the X2 test of independence coherence $2 \mathrm{ch} 3$ tables generated for each pair of control (specifically are 31 pairs, namely comparisons and variable intolerance).

The researcher ran all pairs and reached concrete results which will occur then. Whichever pair does not occur, it is obvious that the statistical findings do not reveal the existence of dependency between the intensity of the disease and the existence of a certain intolerance.

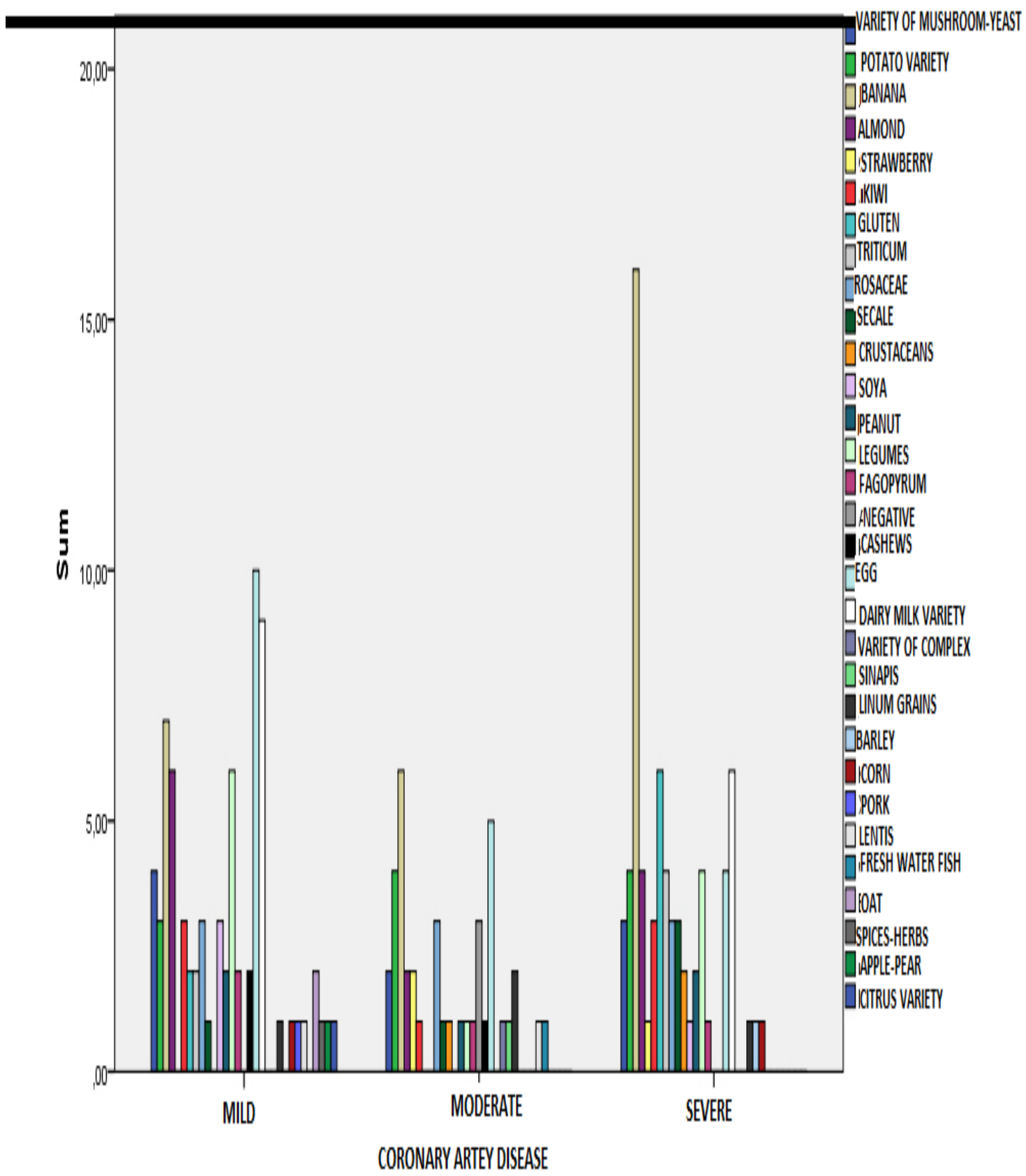

Chart 2. 
Before we begin to analyze the couples mentioned, let's look at the behavior of the disease in relation to gender and age.



Chart 3.

Because age is a scale variable we will implement a different control $\mathrm{x}^{2}$. The test chosen is kruskal wallis (variable is not under normal distribution sig<0.05)

The test shows that the age variable is independent of the intensity of the disease $(\mathrm{sig}>0.05)$. The Boxplot which follow illustrate the test score. However we can claim that older ages typically show higher intensity of the disease. Special attention should be given to the 20th participant, for whom the test recognized him with significant deviant behavior. In particular, while he is young in age, he suffers from the high intensity of the disease. This gives us a suspicion that probably this participant affects the image of the test. Deletion from the sample does not drastically affect the final result, therefore the researcher decided to keep it in the sample.

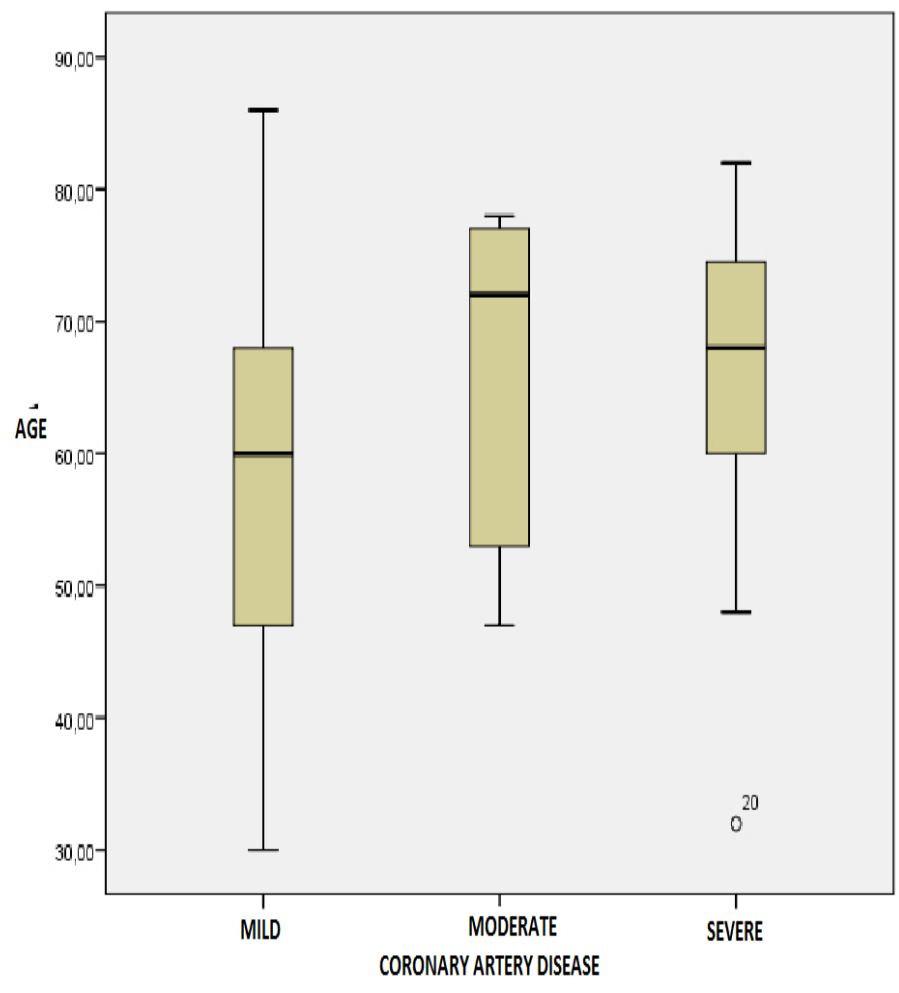

Chart 4. 
Monitoring the statistical tests between the intensity of the disease and intolerances, the author concluded that the majority of intolerances are not related with the intensity of the disease. Only three food intolerances were connected with the intensity and were analyzed below $(\operatorname{sig}<0.05)$

\section{Negative}

Here perhaps we shouldn't mention it at all. Yes the test finds dependence but only 3 people had negative tests.

\section{Egg}

The $\mathrm{x} 2$ test finds that there is some kind of dependency on the two variables.

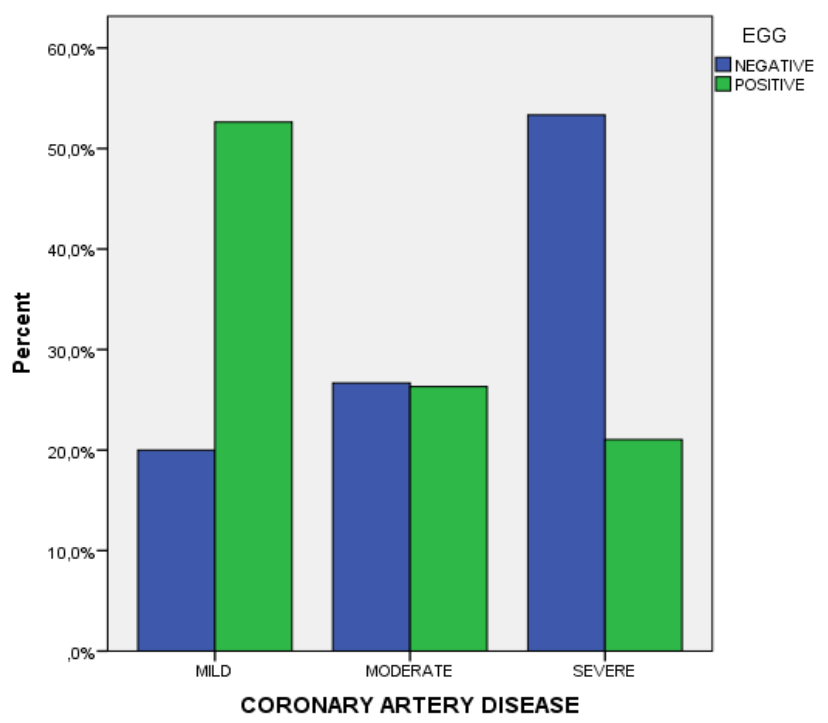

Chart 5.

Indeed, positive intolerance in the egg is connected with the mild intensity of the disease.

\section{Dairy milk variety}

The trend here is that people with intolerance to milk do not experience moderate disease but mostly mild and heavy.

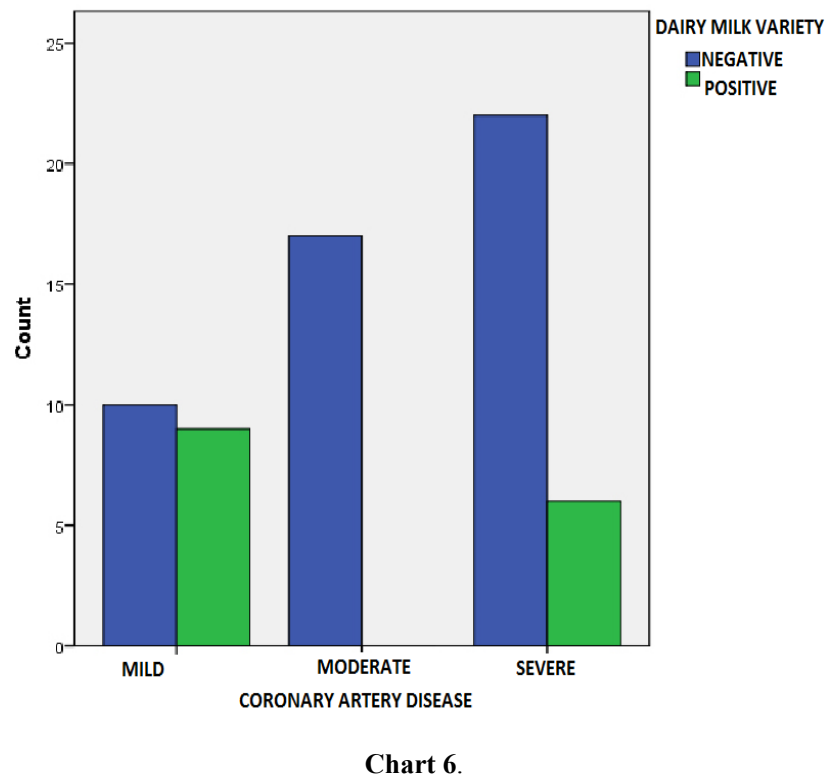

What matters is not the trend but with what intensity the disease connects to any food intolerance. The next graph (7) illustrates only the most popular intolerances, as reflected by the frequency table.

The interpretation of the aforementioned grouped bar chart has the following logic. E.g. banana: $15 \%$ of people with an intolerance to the banana have severe coronary versus $7 \%$ and $6 \%$ of mild and moderate disease, respectively. E.g. fungal percentages are almost the same in all 3 categories.

\section{CONTROL GROUP ANALYSIS}

The data collected from patients and control group (healthy individuals) was refined by the author in order to make the comparisons available for analysis. Therefore the CORONARY ARTERY DISEASE variable recoded into: $0=$ no, $1=$ presence of disease. For the new grouped data, non-parametric test was applied to investigate whether there are statistically significant differences between specific foods (caution: the foods mentioned only in the control group) and the variable of coronary disease.

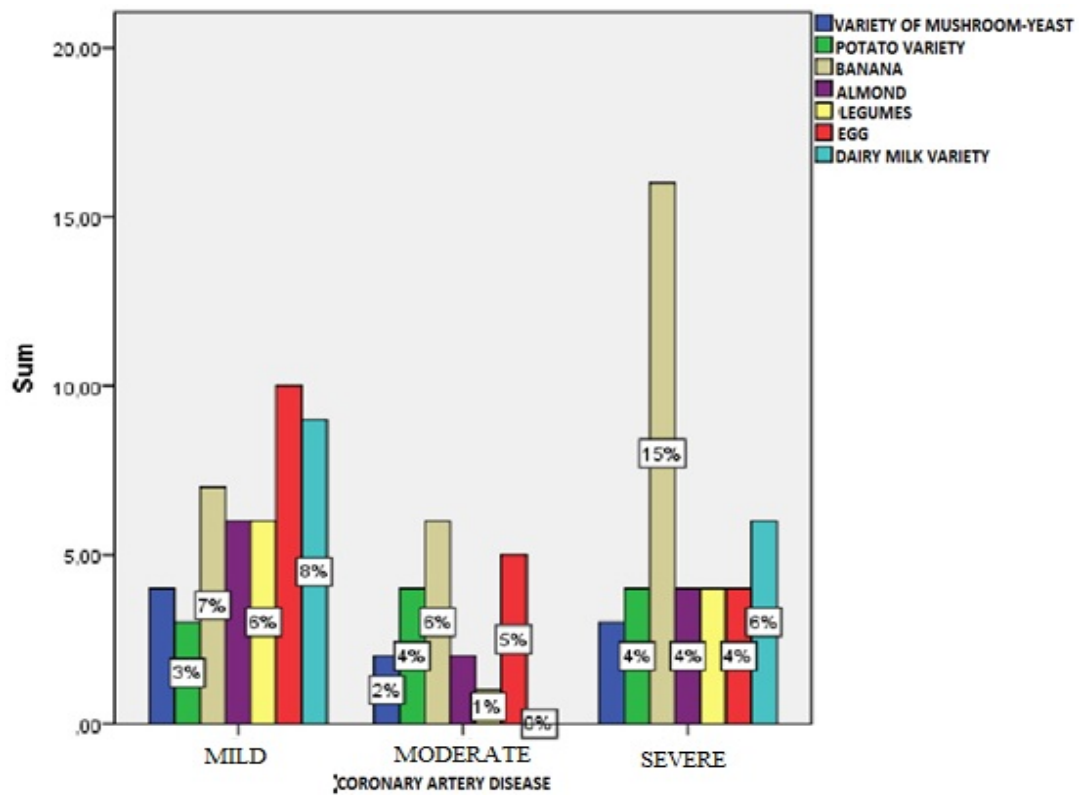

Chart 7. 
Test Statistics ${ }^{\mathrm{a}}$

\begin{tabular}{|l|c|c|c|c|}
\hline & Mann-Whitney U & Wilcoxon W & Z & Asymp. Sig. (2-tailed) \\
\hline POTATO VARIETY & 408,500 & 499,500 &,- 157 &, 875 \\
BANANA & 259,500 & 350,500 & $-2,519$ &, 012 \\
ALMOND & 366,000 & 2446,000 &,- 967 &, 333 \\
TRITICUM & 409,000 & 500,000 &,- 191 &, 848 \\
SECALE & 415,500 & 506,500 &,, 015 &, 263 \\
LEGUMES & 359,500 & 2439,500 &, 848 \\
FAGOPYRUM & 410,000 & 2490,000 & $-1,120$ &, 095 \\
EGG & 315,500 & 2395,500 &,- 191 &, 028 \\
MILK & 289,500 & 2369,500 & $-1,668$ &, 848 \\
LINUM GRAINS & 410,000 & 2490,000 & $-2,198$ &, 208 \\
PORK & 390,500 &,- 191 & $-1,259$ & \\
\hline
\end{tabular}

a. Grouping Variable: coronary disease.

The tests showed differences for the banana and milk. Specifically, while for the rest of the food there was no clear indication to link with the disease, for those two foods was a statistically significant difference in favor of having the disease. CAUTION: the control group was not the best. Teams were uneven and this is shown by the difficulty of the test to confirm the fact that visually there are more differences.

The study of the existence of statistical correlation between food intolerance and heart disease is from a random sample of 75 people and non-cardiac patients, at a ratio of 63:12 without special internal classification on other features.

\section{Discussion}

Generally, medical studies differ depending on whether the purpose of the study is to describe just one population or if we want to understand the relationships between features and identify the causes of a phenomenon (a disease) in a descriptive and explanatory manner. Analogue to this is the statistical analysis used as well as the design of sampling. The descriptive studies are limited to simple descriptors or demographic tables and diagrammatic depiction of data, while the grounds apply statistical tests cases based on biological theories or scenarios.

The descriptive studies done on the population under normal conditions (field studies) present an estimate or fix the prevalence or other characteristics of the population with instantaneous and static character. They provide an overview of the relationships between diseases something that can't be done with hospital surveys. Their advantages are simplicity, the speed with which they can end and their low cost. Their disadvantages include temporal sequence of relationships between variables, the probability of occurrence than the duration of the disease and the inability to study rare diseases because the sample size is usually inadequate.

The explanatory studies aimed at investigating the causality of a disease used sophisticated statistical methods ( $\mathrm{t}$ - test, Wilcoxon test) up to complicated models such as hierarchical generalized linear models and multivariate models. Here include observational studies and interventional studies (clinical trials).

The big problem in statistical science is the fact that, a statistically significant relationship or association between two variables does not automatically imply a real relationship or a causative relationship. This is due to the complexity of the relationships that govern the actual phenomena being examined. Thus the appearance of a phenomenon (e.g. disease) are affected by many factors and therefore the disease relationship - risk factor is influenced by confounders. Even if we exclude these factors, locating actual relations of causality is difficult and escapes from the simple calculation of statistical indicators.

To define causality by statistics, it is necessary to find: consistency, strength, specialty, logical sequence. When a statistically significant correlation satisfies all the criteria above, then we have a strong (though not absolute) indication that this is a causal relationship.

A relationship is called coherent if it appears repeatedly in studies carried out with different designs and different populations. A statistically significant relationship is strong when the effect of the presence of risk factor is great. This effect is usually measured with the proportional increase in the likelihood of death or incidence. A statistically significant relationship is relative if the presence of risk factor leads with high probability to the occurrence of the disease while the absence leads with high probability to absence of the disease. In many cases the statistical analysis does not take into account the temporal sequence in which they appear some potential variables associated with them. Therefore, the risk factor should be under time of the onset of the disease. To have a relationship of logical sequence, it should not contradict proven truths of natural and biological sciences.

In this study we wanted to find a statistically significant relationship that presented the 5 test criteria for deterministic relationship. If a relation is deterministic then you are definitely and statistically significant, independent from the size of the sample. Equivalently, if dependence link does not show statistical significance then surely there is no deterministic relationship between variables under study. To accept, however, the non-existence of statistical significance depends strongly on the type and size of the sample. That is, the sample must be representative and adequate.

The sample that we have here is not accompanied by relevant information on the representativeness and its size of 75 individuals measured. The information that emerged is the following: probably there is no association between intolerance and heart disease. However the sample and the test methodology was not representative enough. 
The conclusions that emerge from the analysis are listed below: no correlation was observed between food intolerance and coronary artery disease, with GOLDEN-based tests.

Most patients had intolerance in banana, egg and milk Intolerance in the variety of potatoes, tomatoes, almond, gluten, wheat, legumes also appear in severe forms of the disease. The analysis of the results is open to two interpretations: the sample of persons was not representative and there were many confounding factors, the methodology was not accurate. It is necessary to develop better diagnostic tools and conduct larger studies that would enlighten the issue of food intolerance association with coronary artery disease. The conclusions of the work does not excludes the association of intolerance to foods with coronary artery disease nor the non-correlation of intolerance with atherosclerosis. We need to understand the process of atherogenesis with more details, so that we can intervene appropriately.

\section{Authors' Contributions}

Angeliki or Aggeliki Tsantsaridou participated in the design of the study and collected all blood samples and drafted the manuscript. Gerasimos Dimisianos carried out the blood tests analyses with golden test and drafted the manuscript. Vissarion-Panagiotis Papathanasiou carried out the statistical analyses of the results and drafted the manuscript.

\section{References}

[1] Dimitrios Tsiantoulas, Cody J. Diehl, Joseph L. Witztum, and Christoph J. Binder. B Cells and Humoral Immunity in Atherosclerosis. Circ Res. 2014 May 23; 114(11): 1743-56.

[2] Blum A1, Peleg A, Weinberg M. Anti-cytomegalovirus (CMV) IgG antibody titer in patients with risk factors to atherosclerosis. Clin Exp Med. 2003 Nov; 3(3):157-60.

[3] Sun YH1, Pei WD, Wu YJ, Wang GG. Zhonghua Yi Xue Za Zhi. Association of herpes simplex virus type2 infection with dyslipidemia in Chinese. 2003 Oct 25;83(20):1774-7. Chinese.

[4] Sundgren NC, Vongpatanasin W, Boggan BM, Tanigaki K, Yuhanna IS, Chambliss KL, Mineo C, Shaul PW. IgG receptor Fc $\gamma$ RIIB plays a key role in obesity-induced hypertension. Hypertension. $2015 \mathrm{Feb}$; 65(2): 456-62.

[5] Gao M, Li Y, Zheng A, Xue X, Chen L, Kong Y. Lymphocyte oxidative stress/genotoxic effects are related to serum IgG and IgA levels in coke oven workers. Scientific World Journal. 2014; 2014 801346.

[6] De Batista PR, Palacios R, Martín A, Hernanz R, Médici CT, Silva MA, Rossi EM, Aguado A, Vassallo DV, Salaices M, Alonso MJ. Toll-like receptor 4 upregulation by angiotensin II contributes to hypertension and vascular dysfunction through reactive oxygen species production. PLoS One. 2014 Aug 5;9(8): e104020.

[7] Kounis NG, Soufras GD, Tsigkas G, Hahalis G. White blood cell counts, leukocyte ratios, and eosinophils as inflammatory marker in patients with coronary artery disease. Clin Appl Thromb Hemost. 2015 Mar; 21(2): 139-43.

[8] Bakirci EM, Demirtas L, Degirmenci H, Topcu S, Demirelli S Hamur H, Buyuklu M, Akbas EM, Ozcicek A, Ozcicek F, Ceyhun G, Topal E. Relationship of the total atrial conduction time to subclinical atherosclerosis, inflammation and echocardiographic parameters in patients with type 2 diabetes mellitus. Clinics (Sao Paulo). 2015 Feb; 70(2): 73-80.

[9] Ceccarelli G1, d'Ettorre G, Mancone M, Francone M, Vullo V. Accelerated coronary atherosclerosis after execution of percutaneous coronary intervention in patient with $\mathrm{HIV} / \mathrm{HCV}$ coinfection: case report and review of the literature. Cardiovasc Revasc Med. 2011 Jul-Aug; 12(4): 262-5.

[10] Miller PS1, Evangelista LS, Giger JN, Martinez-Maza O, Corvera-Tindel T, Magpantay L, Pena G, Doering LV. Exhaustion, immuno-inflammation, and pathogen burden after cardiac surgery: an exploratory study. Eur J Cardiovasc Nurs. 2014 Jun; 13(3): 211-20.

[11] Thomas P. Erlinger, MD, MPH; Edgar R. Miller III, MD, PhD; Jeanne Charleston, RN; Lawrence J. Appel, MD, MPH. Inflammation Modifies the Effects of a Reduced-Fat LowCholesterol Diet on Lipids Results From the DASH-Sodium Trial. Circulation. 2003 Jul 15; 108(2): 150-4.

[12] Elaine Paffen, Moniek P.M. C-reactive protein in atherosclerosis: A causal factor? deMaat j.cardiores. 2006. 03.004 30-39.

[13] Goldschmidt-Clermont PJ1, Dong C, Seo DM, Velazquez OC. Atherosclerosis, inflammation, genetics, and stem cells: 2012 update. Curr Atheroscler Rep. 2012 Jun; 14(3): 201-10.

[14] Mercer JR1, Cheng KK, Figg N, Gorenne I, Mahmoudi M, Griffin J, Vidal-Puig A, Logan A, Murphy MP, Bennett M. DNA damage links mitochondrial dysfunction to atherosclerosis and the metabolic syndrome. Circ Res. 2010 Oct 15; 107(8): 1021-31.

[15] Bagnato C1, Thumar J, Mayya V, Hwang SI, Zebroski H, Claffey KP, Haudenschild C, Eng JK, Lundgren DH, Han DK. Proteomics analysis of human coronary atherosclerotic plaque: a feasibility study of direct tissue proteomics by liquid chromatography and tandem mass spectrometry. Mol Cell Proteomics. 2007 Jun; 6(6): 1088-102

[16] Sanner JE1, Frazier L. The role of serotonin in depression and clotting in the coronary artery disease population. J Cardiovasc Nurs. 2011 Sep-Oct; 26(5): 423-9.

[17] Lüscher TF1. Thrombocyte-vascular wall interaction and coronary heart disease. Schweiz Med Wochenschr. 1991 Dec 28; 121(5152): 1913-22. Review. German. PMID: 1763300.

[18] Demirtürk M1, Polat N, Güz G, Gürdal A, Altun I, Gelincik A Toz B, Oflaz H, Colakoğlu B, Dal M, Büyüköztürk S. There is an increased risk of atherosclerosis in hereditary angioedema. Int Immunopharmacol. 2012 Jan; 12(1): 212-6.

[19] Theoharides TC Clin Ther. Is a subtype of autism an allergy of the brain? 2013 May; 35(5): 584-91.

[20] Eelen G, de Zeeuw P, Simons M, Carmeliet P. Endothelial cell metabolism in normal and diseased vasculature. Circ Res. 2015 Mar 27; 116(7): 1231-44.

[21] Kim YW1, Byzova TV. Oxidative stress in angiogenesis and vascular disease. Blood. 2014 Jan 30; 123(5): 625-31.

[22] Greenberg DA1, Jin K. Vascular endothelial growth factors (VEGFs) and stroke. Cell Mol Life Sci. 2013 May; 70(10): 1753-61.

[23] Tetè S, Tripodi D, Rosati M, Conti F, Maccauro G, Saggini A, Salini V, Cianchetti E, Caraffa A, Antinolfi P, Toniato E, Castellani ML, Pandolfi F, Frydas S, Conti P, Theoharides TC. Endothelial cells, cholesterol, cytokines, and aging. Int $\mathrm{J}$ Immunopathol Pharmacol. 2012 Apr-Jun; 25(2):355-63.

[24] Owczarek A1, Babińska M, Szyguła-Jurkiewicz B, Chudek J. Chronic inflammation in patients with acute coronary syndrome and chronic kidney disease. Kardiol Pol. 2011; 69(4): 388-93.

[25] Virdis A1, Schiffrin EL. Vascular inflammation: a role in vascular disease in hypertension? Curr Opin Nephrol Hypertens. 2003 Mar; 12(2): 181-7.

[26] Anna Rybak, Bożena Cukrowska, Jerzy Socha, and Piotr Socha. Long Term Follow Up of Celiac Disease-Is Atherosclerosis a Problem? Nutrients. 2014 Jul 21; 6(7): 2718-29.

[27] Saeed F1, Pasha I, Anjum FM, Sultan MT. Arabinoxylans and arabinogalactans: a comprehensive treatise. Crit Rev Food Sci Nutr. 2011 May; 51(5): 467-76.

[28] Thiel C1. Food allergies and intolerance reactions. Z Ernahrungswiss. 1991 Sep; 30(3): 158-73.

[29] Sarzi-Puttini P1, Atzeni F, Carrabba M. Cardiovascular risk factors in systemic lupus erythematosus and in antiphospholipid syndrome. Minerva Med. 2003 Apr; 94(2): 63-70.

[30] Storch H, Reuter W Z. Immune reactivity in patients with disorders of lipid metabolism. Gesamte Inn Med. 1981 Apr 1; 36(7): 289-92

[31] Beaumont JL, Berard M, Antonucci M, Delplanque B, Vranckx R. Inhibition of lipoprotein lipase activity by a monoclonal immunoglobulin in autoimmune hyperlipidemia. Atherosclerosis. 1977 Jan; 26(1): 67-77. 
[32] James TG Li M.D.. What is the difference between a food intolerance and food allergy? June 3, 2011, Mayo Clinic, Mayo Foundation for Medical Education and Research. Accessed November $9^{\text {th }} 2013$.

[33] Ho MH, Wong WH, Chang C. Clinical spectrum of food allergies: a comprehensive review. Clin Rev Allergy Immunol. 2014 Jun; 46(3): 225-40.

[34] Genuis SJ. Sensitivity-related illness: the escalating pandemic of allergy, food intolerance and chemical sensitivity. Sci Total Environ. 2010 Nov 15; 408(24): 6047-61.

[35] Review article: the aetiology, diagnosis, mechanisms and clinical evidence for food intolerance. Aliment Pharmacol Ther 2014 Dec 3.

[36] Ohtsuka Y. Food intolerance and mucosal inflammation. Paediatr Int. 2015 Feb; 57(1): 22-29.

[37] Croca S, Bassett P, Chambers S, Davari M, Alber KF, Leach O, Ioannou Y, Giles I, Isenberg D, Rahman A. IgG anti-apolipoprotein A-1 antibodies in patients with systemic lupus erythematosus are associated with disease activity and corticosteroid therapy: an observational study. Arthritis Res Ther. 2015 Feb 9; 17(1): 26

[38] Montecucco F, Braunersreuther V, Burger F, Lenglet S, Pelli G, Carbone F, Fraga-Silva R, Stergiopulos N, Monaco C, Mueller C, Pagano S, Dallegri F, Mach F, Vuilleumier N. Anti-apoA-1 auto-antibodies increase mouse atherosclerotic plaque vulnerability, myocardial necrosis and mortality triggering TLR2 and TLR4. Thromb Haemost. 2015 Apr 16; 114(20150416). [Epub ahead of print] PMID:25879306.

[39] Caselli M, Zuliani G, Cassol F, Fusetti N, Zeni E, Lo Cascio N, Soavi C, Gullini S. Test-based exclusion diets in gastroesophageal reflux disease patients: a randomized controlled pilot trial. World J Gastroenterol. 2014 Dec 7; 20(45): 17190-5.

[40] Signs of microbial sensibilization in patients with various cardiovascular diseases. Zh Mikrobiol Epidemiol Immunobiol. 2015 Jan-Feb; (1): 75-81.

[41] Pietschmann N. Food Intolerance: Immune Activation Through Diet-associated Stimuli in Chronic Disease. Altern Ther Health Med. 2015 Jul; 21(4): 42-52.

[42] Merched AJ1, Chan L. Nutrigenetics and nutrigenomics of atherosclerosis. Curr Atheroscler Rep. 2013 Jun; 15(6): 328

[43] Skibska B, Goraca A. The Protective Effect of Lipoic Acid on Selected Cardiovascular Diseases Caused by Age-Related Oxidative Stress. Oxid Med Cell Longev. 2015;2015:313021. Epub 2015 Apr 8. Review. PMID: 25949771

[44] Pietschmann N. Food Intolerance: Immune Activation Through Diet-associated Stimuli in Chronic Disease. Altern Ther Health Med. 2015 Jul; 21(4): 42-52.

[45] Fritscher-Ravens A, Schuppan D, Ellrichmann M, Schoch S, Röcken C, Brasch J, Bethge J, Böttner M, Klose J, Milla PJ. Confocal endomicroscopy shows food-associated changes in the intestinal mucosa of patients with irritable bowel syndrome. Gastroenterology. 2014 Nov; 147(5): 1012-20. e4.

[46] Cuomo R, Andreozzi P, Zito FP, Passananti V, De Carlo G, Sarnelli G. Irritable bowel syndrome and food interaction. World J Gastroenterol. 2014 Jul 21; 20(27): 8837-45.

[47] Skibska B, Goraca A. The Protective Effect of Lipoic Acid on Selected Cardiovascular Diseases Caused by Age-Related Oxidative Stress. Oxid Med Cell Longev. 2015; 2015: 313021. Epub 2015 Apr 8.

[48] Merched AJ. Nutrigenetics and nutrigenomics of atherosclerosis Chan L. Curr Atheroscler Rep. 2013 Jun; 15(6): 328.

[49] Kotlyar DS, Shum M, Hsieh J, Blonski W, Greenwald DA Non-pulmonary allergic diseases and inflammatory bowel disease: a qualitative review. World J Gastroenterol. 2014 Aug 28; 20(32): 11023-32.

[50] Vojdani A. Detection of IgE, IgG, IgA and IgM antibodies against raw and processed food antigens. Nutr Metab (Lond). 2009 May 12; 6: 22.

[51] Palermo A1, Maggi D, Maurizi AR, Pozzilli P, Buzzetti R. Prevention of type 2 diabetes mellitus: is it feasible? Diabetes Metab Res Rev. 2014 Mar; 30 Suppl 1: 4-12.
[52] Mansueto P, D'Alcamo A, Seidita A, Carroccio A. Food allergy in irritable bowel syndrome: The case of non-celiac wheat sensitivity World J Gastroenterol. 2015 Jun 21; 21(23): 7089-109.

[53] Green PH, Lebwohl B, Greywoode R. Celiac disease. J Allergy Clin Immunol. 2015 May; 135(5): 1099-1106.

[54] Parfenov AI, Akhmadullina OV, Sabel'nikova EA, Belostotskii NI, Gudkova RB, Khomeriki SG. Carbohydrase activities may serve as a marker for small intestinal mucosal recovery in patients with celiac disease. Ter Arkh. 2015; 87(2): 24-9.

[55] Ohtsuka Y. Food intolerance and mucosal inflammation. Paediatr Int. $2015 \mathrm{Feb}$; 57(1): 22-29.

[56] Lomer MC. Review article: the aetiology, diagnosis, mechanisms and clinical evidence for food intolerance. Aliment Pharmacol Ther. 2015 Feb; 41(3): 262-75.

[57] Frehn L, Jansen A, Bennek E, Mandic AD, Temizel I, Tischendorf S, Verdier J, Tacke F, Streetz K, Trautwein C, Sellge G. Distinct patterns of IgG and IgA against food and microbial antigens in serum and feces of patients with inflammatory bowel diseases. PLoS One. 2014 Sep 12; 9(9): e106750.

[58] Wilson $\mathrm{K}$. The role of food intolerance in functional gastrointestinal disorders in children. Hill RJ Aust Fam Physician. 2014 Oct; 43(10): 686-9.

[59] Di Cerbo A, Canello S, Guidetti G, Laurino C, Palmieri B5. Unusual antibiotic presence in gym trained subjects with food intolerance; a case report. Nutr Hosp. 2014 Aug 1; 30(2): 395-8.

[60] Vorozhko IV, Sentsova TB, Kirillova OO, Gapparova, Chekhonina IuG. Soluble apoptosis markers in obese patients with food intolerance. Vopr Pitan. 2014; 83(2): 22-6.

[61] Lomer MC. Review article: the aetiology, diagnosis, mechanisms and clinical evidence for food intolerance. Aliment Pharmacol Ther. $2015 \mathrm{Feb}$; 41(3): 262-75.

[62] Lik Sprava. The link between food intolerance and weight: a new perspective in the treatment of obesity. Rotter MM. 2014 Jan-Feb; (1-2): 87-91. Ukrainian. PMID: 24908967.

[63] Storch H, Reuter W Z. Immune reactivity in patients with disorders of lipid metabolism. Gesamte Inn Med. 1981 Apr 1; 36(7): 289-92

[64] Beaumont JL, Berard M, Antonucci M, Delplanque B, Vranckx R. Inhibition of lipoprotein lipase activity by a monoclonal immunoglobulin in autoimmune hyperlipidemia. Atherosclerosis. 1977 Jan; 26(1): 67-77.

[65] Okamoto Y1, Tominaga K, Uemura S, Matsuoka H, Tsujii T, Nakano H. Hypertriglyceridemia caused by the autoantibody to lipases for plasma lipoproteins: a case report. J Atheroscler Thromb. 1995; 2(1): 66-9.

[66] Sarzi-Puttini P, Atzeni F. Cardiovascular risk factors in systemic lupus erythematosus and in antiphospholipid syndrome. Carrabba M. Minerva Med. 2003 Apr; 94(2): 63-70.

[67] Paula A. Hayes, BSc, Marianne H. Fraher, MB, MRCPI, FRCPath, and Eamonn M. M. Quigley, MD, FRCP, FACP, FACG, Irritable Bowel Syndrome: The Role of Food in Pathogenesis and Management. FRCPI corresponding author Gastroenterol Hepatol (N Y). 2014 Mar; 10(3): 164-74.

[68] Sentsova TB, Vorozhko IV, Isakov VA, Morozov SV, Shakhovskaia AK. Immune status estimation algorithm in irritable bowel syndrome patients with food intolerance. Eksp Klin Gastroenterol. 2014; (7):13-7. Russian.

[69] [Article in Chinese] Liu XJ, Zhu TT, Zeng R, Chang L, Li FY, Li WS, Jiang YM. An epidemiological study of food intolerance in 2434 children. Zhongguo Dang Dai Er Ke Za Zhi. 2013 Jul; 15(7): 550-4. Chinese.

[70] Anna Rybak, Bożena Cukrowska, Jerzy Socha, and Piotr Socha. Long Term Follow Up of Celiac Disease-Is Atherosclerosis a Problem? Nutrients. 2014 Jul 21; 6(7): 2718-29.

[71] Christopher T. Chan, Maggie Lieu, Ban-Hock Toh, Tin S. Kyaw, Alexander Bobik, Christopher G. Sobey, and Grant R. Drummond. Antibodies in the Pathogenesis of Hypertension. Biomed Res Int. 2014; 2014: 504045. 